\title{
Unconventional Non-Invasive Diagnostic Techniques and Treatment of White Spot Lesions in Paediatric Dentistry and Orthodontics
}

\author{
Ogodescu Alexandru (Assoc. Professor, PhD), \\ Igna Andreea (Postgraduate Student), \\ Ogodescu Emilia (Assis. Professor, PhD), \\ Luca Magda (Assis. Professor, PhD)
}

Department of Paediatric Dentistry, Faculty of Dental Medicine, University of Medicine and Pharmacy "Victor Babeş", Timişoara, ROMANIA

doi: 10.19044/esj.2017.v13n15p339 URL:http://dx.doi.org/10.19044/esj.2017.v13n15p339

\begin{abstract}
Minimal Intervention Dentistry (MID), a concept that aims to minimize the interventional procedures applied on teeth affected by dental caries, emphasizes the importance of prevention and detection of lesions in early stage. The white spot lesion (WSL) is the first visible stage of the carious process, affecting the enamel. Their detection and treatment has been significantly improved in the last decade through new technology available. Our paper describes a non-invasive detection method, using a lighttransmission device (transillumination of the teeth using Diagnocam, by Kavo), combined with a resin infiltration technique (using Icon, by DMG) of the WSL.
\end{abstract}

Keywords: MID, non-invasive, WSL, conservative

\section{Introduction}

Dental caries is the most prevalent of the oral diseases worldwide. The dental profession currently is faced with an enormous task of how to manage the high burden of consequences of the caries process amongst the world population. A new approach on the management of carious lesion development and its progression was published by FDI World Dental Federation on MID in 2000- the Minimal Intervention Dentistry (MID) (Frencken et al., 2012). Early detection and diagnosis of the carious lesion are a primary consideration of the MID concept (Tassery \& Manton, 2016), since it could give the opportunity to reverse the process and eliminate or at least postpone the surgical treatment (Marinova-Takorovaa, 2016). Another 
aspect of the MID philosphy and a goal of modern dentistry is to manage non-cavitated caries lesions non-invasively through remineralization in an attempt to prevent disease progression (Bilgin, 2016). However, for such a treatment approach to function in general dental practice, the dentist needs access to methods which allow not only detection of early lesions but also monitoring of the effect of interventions, that is, to observe progression, arrest, or regression of such lesions over time (Astvaldsdottir et al., 2012). The first stage in carious lesion emergence is represented by the White Spot Lesion (WSL). This is also the moment when therapeutical measures should be taken. Unlike the classical approach of removing the incipient demineralised lesion, the present technology, developed by DMG company from Germany, allows keeping the hard dental structure and its reinforcement by infiltration.

A white spot lesion (WSL) can be defined as the pre-cavitation stage of a carious lesion. Internationally used systems for classifying the stages of progression of dental caries recognize a pre-white spot lesion which is not visible when the enamel surface is wet, but can be seen once the overlying plaque is removed and the tooth surface is dried with compressed air. Prewhite spot lesions can also be detected using a number of fluorescence systems. The further progression of this pre-white spot lesion leads to a lesion which is detectable by the clinician on a wet enamel surface without the aid of magnification (the WSL). Whilst its macroscopic surface structure remains intact, remineralization of such lesions can be a powerful means for preventing their cavitation (Walsh \& Brostek, 2013).

In pediatric patients, WSL are a common finding. A high prevalence of tooth enamel white spots was found in children up to 36 months old, mainly associated with age group, duration of bedtime milk feeding habit, age of initial practice of oral hygiene and presence of caries lesions with cavitation (Pagliari Tiano et al., 2009). Particularly exposed to the development of caries are the proximal surfaces of the posterior teeth. In the primary dentition, in regard to the first molars, these are mainly the distal contact surfaces, and, to a lesser extent, the mesial contact surfaces (WojcikChecinska, 2015).

WSL associated with fixed orthodontic appliances are a common adverse effect of orthodontic treatment., a problem that can be minimized with appropriate prevention, management, and treatment approaches (Heymann \& Grauer, 2013). The occurence of WSL can be attributed to the difficulties in performing oral hygiene procedures on bonded dental arches and the prolonged plaque accumulation on tooth surfaces, which lead to a decrease in $\mathrm{pH}$ that tips the demineralization-remineralization balance toward mineral loss (demineralization), which in turn can lead to WSL 
development and eventually to cavitation and caries extending into the dentin (Sundararaj et al., 2015).

Classifications of white spot lesions (WSL), which may include several different criteria, are useful in applying the appropriate treatment. There are real WSL and pseudo-WSL. The real ones are produced directly on the enamel surface, as a consequence of repeated acid agression, while the pseudo-WSL only imitate the white mate aspect (different forms of amelogenesis imperfecta). Based on etiology, they can be exogenous - when an outside agent is causing the lesion, and endogenous - when the causative factor acts from the inside, during the enamel formation stage. According to their localization, WSL can be vestibular, proximal and combined vestibular and proximal, situated in the gingival 1/3 (usually the real ones), the medium 1/3 (for the most part after orthodontic treatment) or the incisal 1/3 (different types of amelogenesis imperfecta or Molar-IncisorHypomineralisation syndrome ) of the dental surface. WSL can be associated to orthodontic treatment or not. According to this proposed classification, the lesions in the images below can be described as follows:

$>$ Real WSL - exogenous, situated in the vestibular area, in the gingival 1/3, unassociated to orthodontic treatment - it is practically the aspect of a classical WSL (Fig.1)

$>$ Real WSL - exogenous, situated in the vestibular and proxiaml area, in the medium $1 / 3$, associated to orthodontic treatament - it is a WSL that emerged post-orthodontic treatment (Fig.2).

$>$ Pseudo-WSL, endogenous, situated in the vestibular area, in the incisal $1 / 3$, unassociated to orthodontic treatment - it is practically a hypomineralised form of the amelogenesis imperfecta, which imitates a WSL (Fig.3).

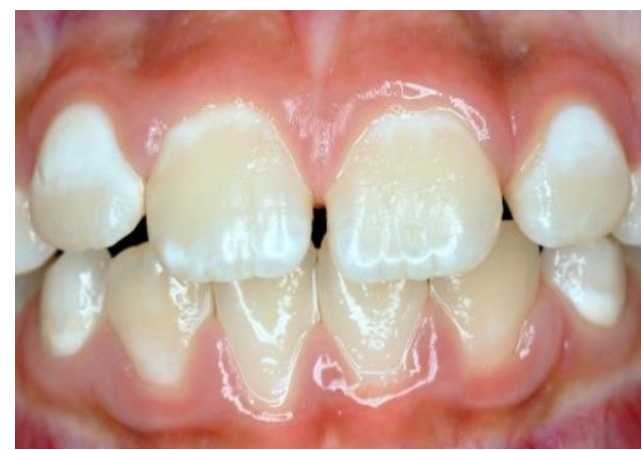

Fig.1 Clinical aspect of a classical WSL 


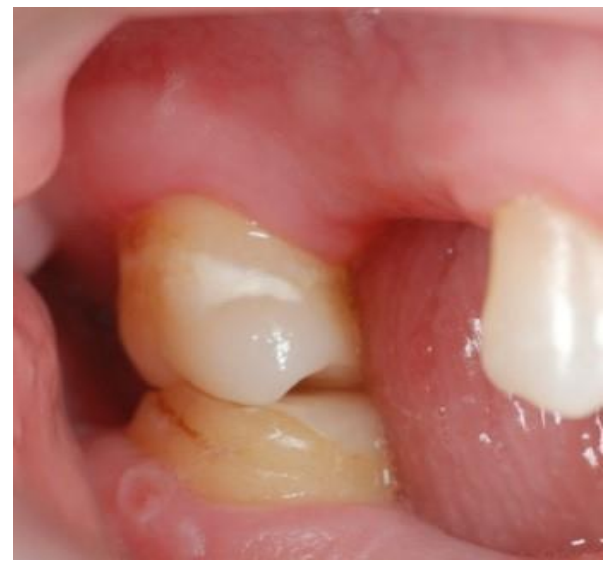

Fig.2 Clinical aspect of a WSL associated to orthodontic treatment

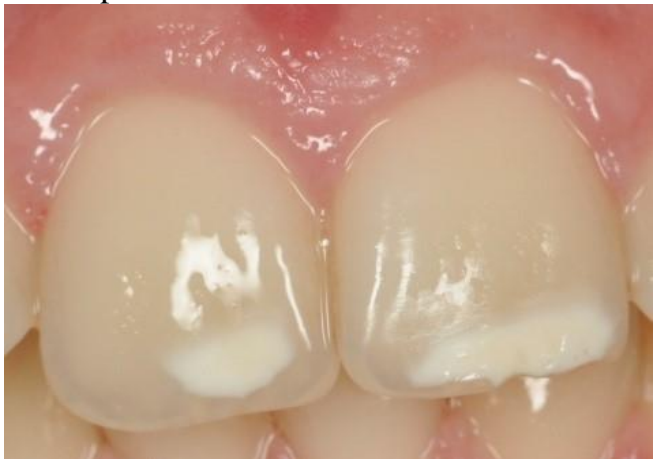

Fig.3 Hypomineralised form of the amelogenesis imperfecta in the incisal $1 / 3$ of the vestibular surface, which imitates a WSL

The infiltration therapy is basically a strong indication for clinical cases shown in Fig.1 and Fig.2, and a limited indication in the case from Fig.3. Practitioners try to extend this method though to clinical situations that resemble the case from Fig.3, by repeating the acid attack stage for 3-4 times, aiming to open the lesion and mask the esthetic deffect by infiltration. To achieve the desired results, a good clinical experience in infiltration therapy is necessary. All the same, the results are still sometimes incomplete (Fig.4).
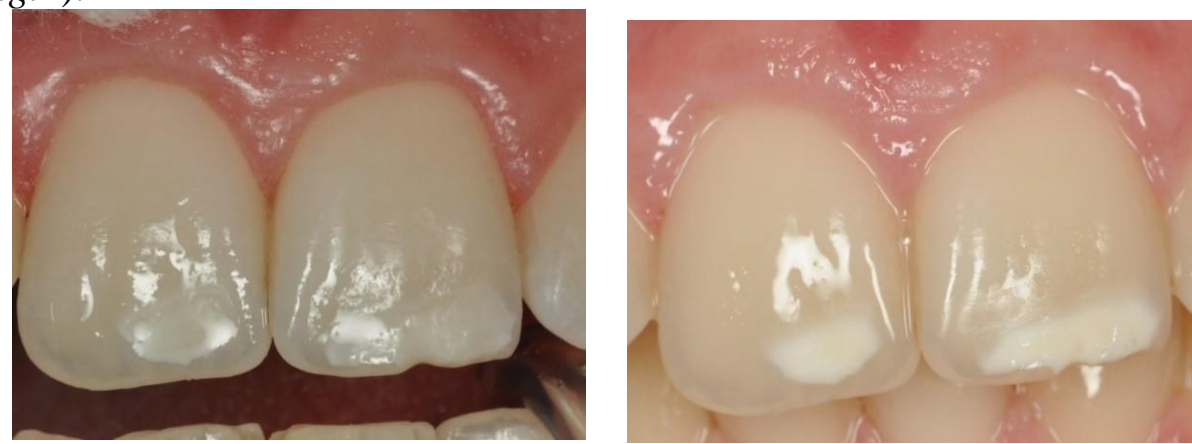

Fig. 4 Pseudo-WSL: initial clinical aspect (right) and the almost-complete masking of the deffect (left) 
The success of the infiltration therapy depends essentially on an adequate opening of the lesion during the acid attack stage. Wether this was obtained, can be evalluated in the drying stage, immediatly after the drying agent was applied. The lesion should have the same colour as the tooth. If white areas are still present, the lesion is not completely open and thus repeating the acid attack is neccessary.

Lesion detection implies an objective method of determining whether or not the disease is present, lesion assessment aims to characterize once it has been detected and caries diagnosis implies a human professional summation of all available data (Gomez, 2014). Traditionally, visual,tactile and radiographic methods are used to detect carious lesion (Tassery \& Manton, 2016). An International Consensus Workshop on Caries Clinical Trials (ICW-CCT) (Pitts, 2004) stated visual detection as the standard of caries diagnosis. A limitation of this method is its subjectivity, which can be improved though, by the use of indices such as International Caries and Detection and Assessment System (ICDAS) (Tassery \& Manton, 2016). Another limitation is the difficulty encountered in detecting caries on the proximal surfaces of the posterior teeth because of their specific location in the oral cavity (Blazejewska et al., 2016). Tactile detection, especially using a sharp explorer, is not recommended (Tassery \& Manton, 2016). Radiography and radiovisiography (RVG) are the most common additional methods of caries detection. RVG has significantly reduced the dose of Xrays, and the image taken digitally can be stored, processed and replicated many times (Blazejewska et al., 2016). But in children and teenagers, obtaining satisfactory bitewings is often problematic and sometimes impossible. Overlapping effects on the radiograph and fixed orthodontic appliances make the detection of primary lesions more difficult. Furthermore, the indication and repetability of dental x-rays are considerably limited because of the associated ionizing radiation (Sochtig et al 2014). However, progress in technology has led to the development of new additional, non-invasive methods of carious lesion detection, using lighttransmission, electrical impedance or photothermal radiometry. Lighttransmission devices vary according to the emission-principle used, namely: Laser Fluorescence (LF or Laser Induced Fluorescence-LIF), LED Fluorescence, QLF (Quantitative Light-induced Fluorescence) and FibreOptic Transillumination (FOTI) at a near-infrared (NIR) wavelength. They analyse the fluorescence reflected from the dental tissues, providing information about the area and extent of the lesion, expressed either as numerical scales or digital images (with the aid of a CCD camera and a computer software).

The Digital Imaging Fiber- Optic Transillumination (DIFOTI) system, a refinement of its predecessor, FOTI, was designed to overcome the 
limitations of FOTI by providing digital image capture. Such images can be stored in digitized form and compared with previously acquired images. The major advantage of the method is that it is noninvasive and can therefore be as frequently used as needed (Astvaldsdottir et al., 2012).

The DIAGNOcam ${ }^{\circledR}(\mathrm{KaVo}$ - Biberach, Germany) - Fig. 5,6 is an intraoral camera based on a slight modiffication of the DIFOTI technology. The broad spectrum illumination source used in FOTI technique (the photopolymerization lamp, for instance) has been replaced by a narrow band light source in the near-infrared spectrum, which allows deeper penetration within the tissues (van der Veen, 2015). It consists of a camera for NIR light emission connected with a USB port to a computer and specific software. Two terminals with lateral optical fibres transmit the light through the gum and alveolar bone to the dental root and then up to the crown. In cases of a carious lesions, the light is scattered and reduced via a mechanism similar to FOTI and DIFOTI but within a differentlight range (700 to $1500 \mathrm{~nm}$ ), resulting in deeper tissue penetration (Russotto et al., 2016).

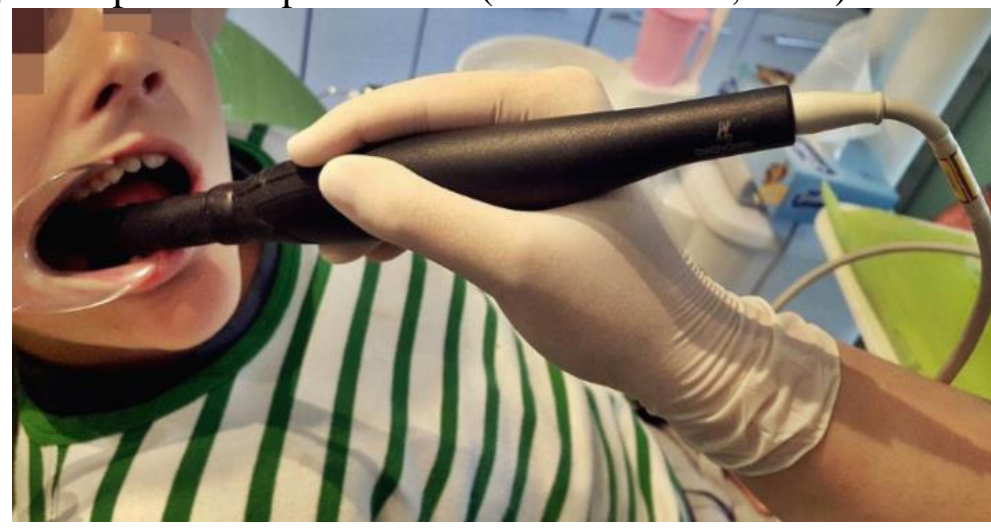

Fig. 5 The DIAGNOcam ${ }^{\circledR}$ intraoral camera

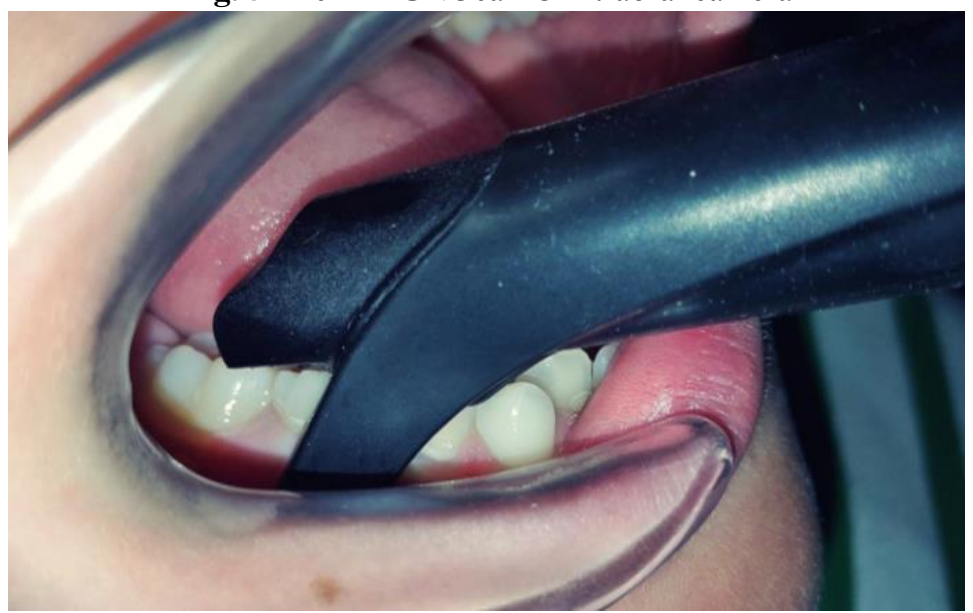

Fig. 6 The DIAGNOcam ${ }^{\circledR}$ terminal with lateral optical fibres 
Healthy enamel has a light gray shade, while the dentine gives a darker gray shade. Areas with changed light transmission, e.g. enamel cracks, fillings or carious lesions (Fig. 7), are visible as darker or lighter lines and areas of various shapes, standing out from the regular structure (Błażejewska et al., 2016).

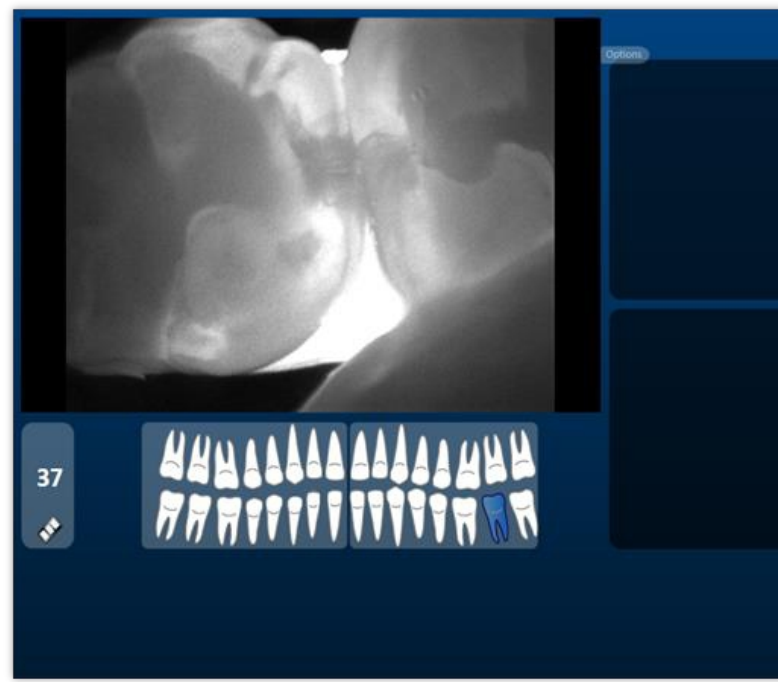

Fig. 7 DIAGNOcam image (within software) of proximal lesions on teeth 3.6 and 3.7

Clinical and in vitro studies revealed the efficiency of DIAGNOcam system in detecting proximal non-cavitated carious lesions, which makes it a viable method for use in addition to visual inspection, as a replacement for the bite-wing x-rays (Astvaldsdottir et al., 2012; Błażejewska et al., 2016 and Marinova-Takorovaa, 2016). It seems to perform in a comparable, and sometimes even better, manner than RE in the diagnosis of interproximal caries of non-restored teeth. In particular, this diagnostic device could be used to monitor caries restricted to enamel over time. Moreover, it could be particularly useful in patients who should not be exposed to ionizing radiation unless absolutely necessary (e.g., pregnant women and children) (Russotto et al 2016). DIFOTI is more accurate compared to bitewing radiography because the extension of tooth structure demineralization can be more precise to evaluate and helps in determining the treatment course, noninvasive approach through remineralisation or minimally-invasive intervention (Cirligeriu et. al 2015).

The infiltration technique implies going through the following steps:

$>$ professional cleaning of the tooth followed by air dry

Note: the teeth can be distanced a little from each other (1-3 days prior to the infiltration) using an orthodontic elastic separating ring, to ease the access (Fig. 8) 


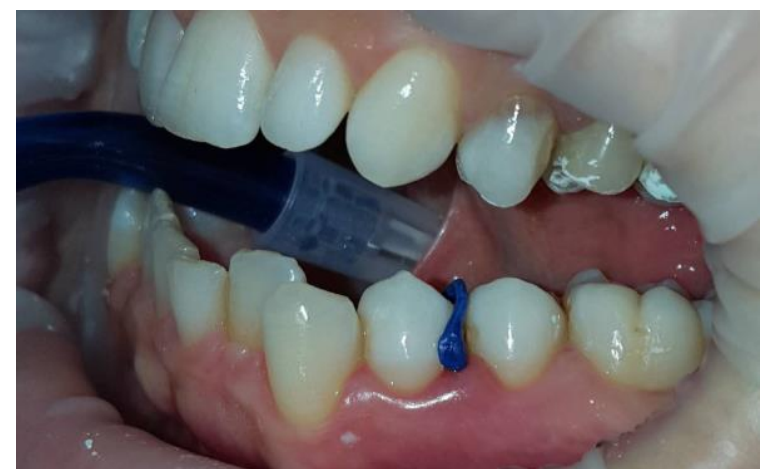

Fig. 8 Gaining adequate interdental space with an orthodontic elastic separating ring

$>\quad$ isolation of the tooth (MiniDam by DMG) - Fig.9

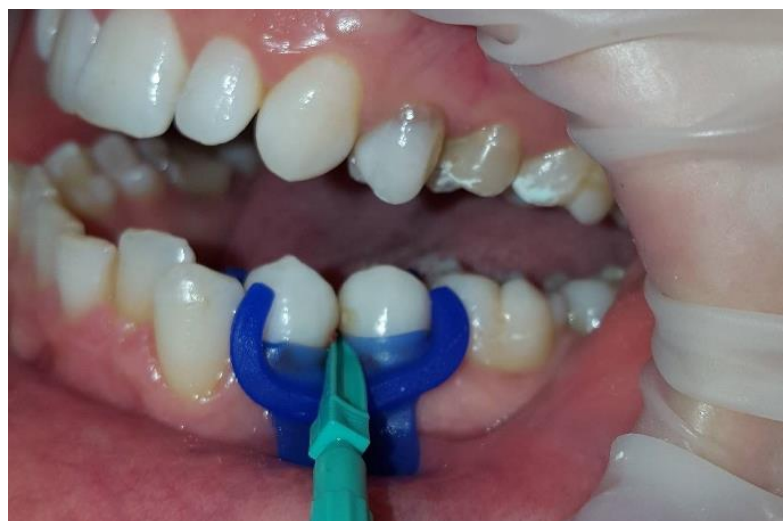

Fig. 9 Isolation of the tooth with MiniDam and dental wedge

$>$ applying the conditioning agent - Icon Etch, for 2 minutes, with the aid of the special proximal tip (Fig.10)

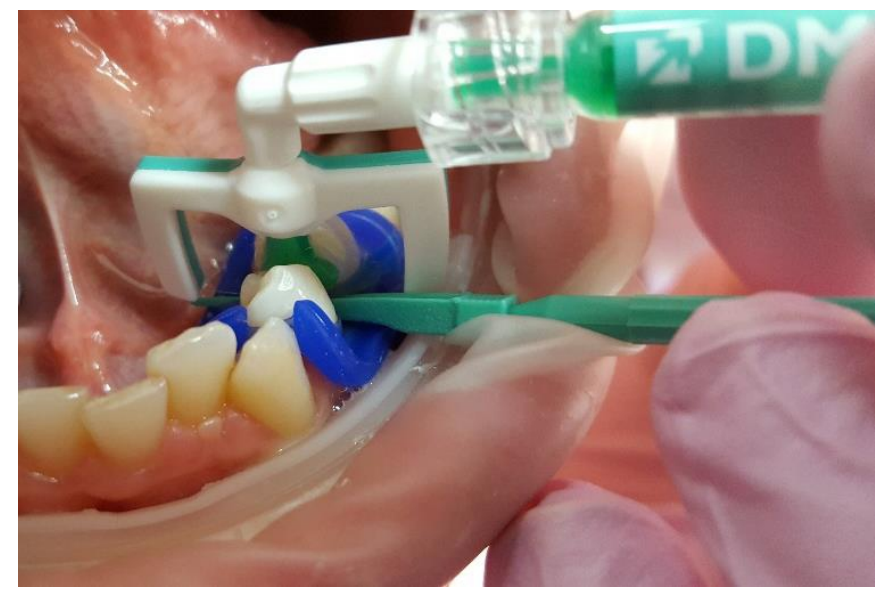

Fig. 10 Icon Etch application with the approximal tip, the green side facing the white spot lesion 
> water rinsing for 30 seconds and thorough air drying (Fig. 11)

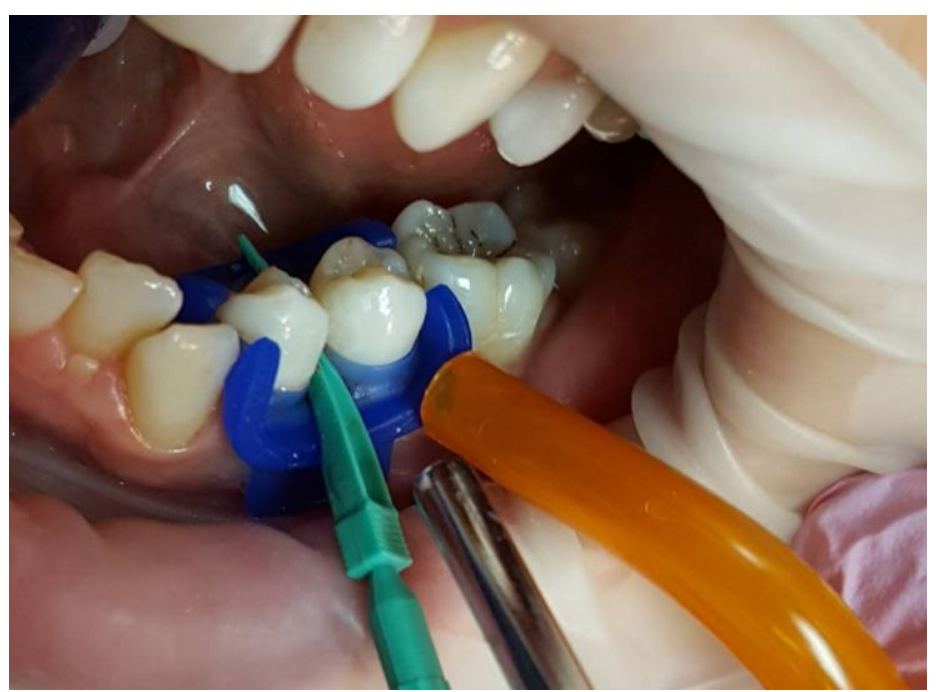

Fig. 11 Washing and drying the white spot lesion

applying the drying agent - Icon Dry for 30 seconds (Fig. 12) and air drying for 30 seconds (Fig.13)

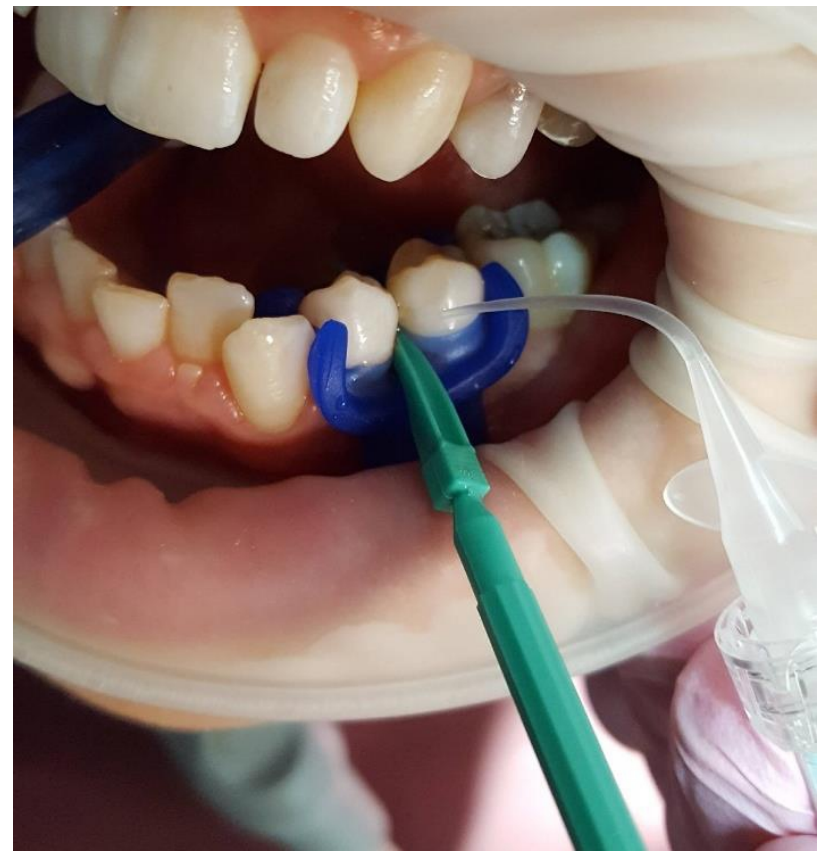

Fig, 12 Icon-Dry for 30 seconds; at this point the completely open WSL will have the color of the around enamel 


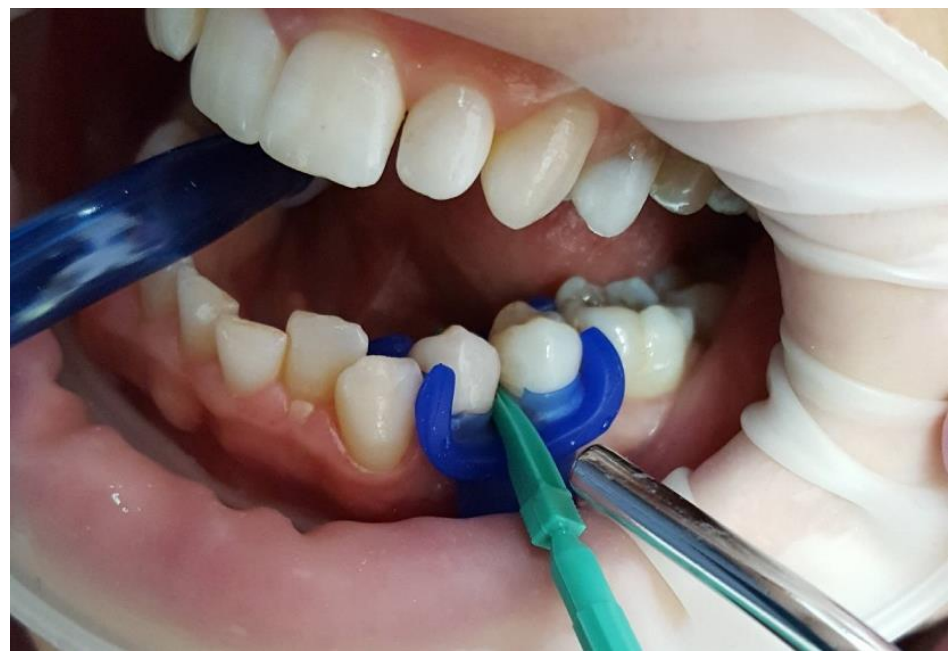

Fig. 13 Air drying of the WSL - the lesion will become whitish

Note: floss is applied for removal of excess material (Fig.14)

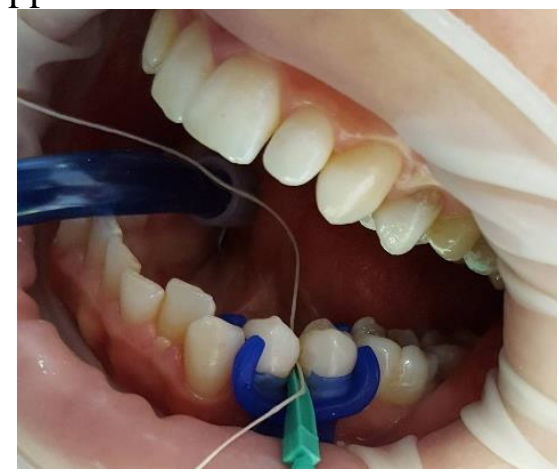

Fig. 14 Dental floss for excess removal

$>$ Icon Infiltrant is applied through the proximal tip, for 3 minutes (Fig.15), slightly air-dryed, then polymerised for 40 seconds (Fig. 16)

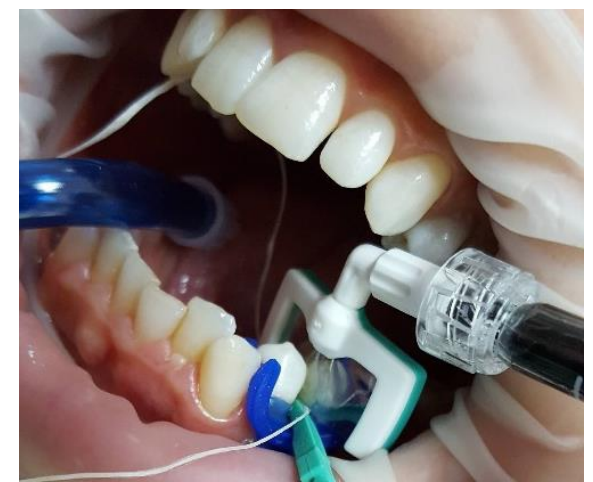

Fig. 15 Application of the Icon Infiltrant with the approximal tip 


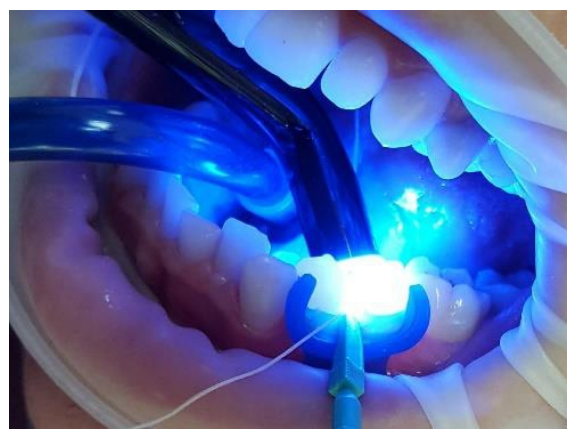

Fig. 16 Photopolimerisation of infiltration

$>$ finally the tooth is polished to smoothen the surface (Fig. 17)

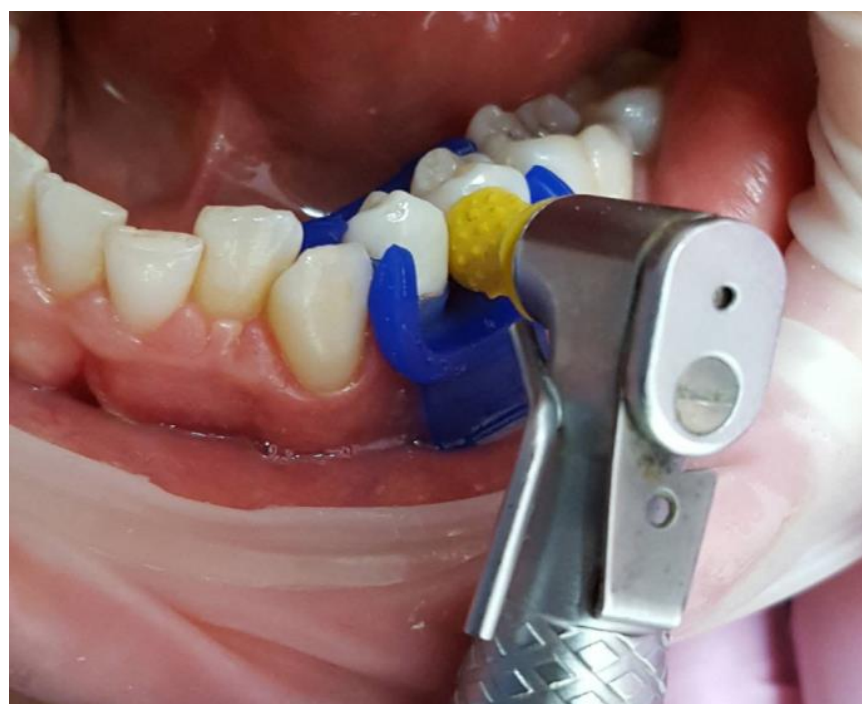

Fig. 17 Removal of infiltrating debris and surface finishing

$>$ the final aspect of the infiltrated lesion can be seen in Fig. 18

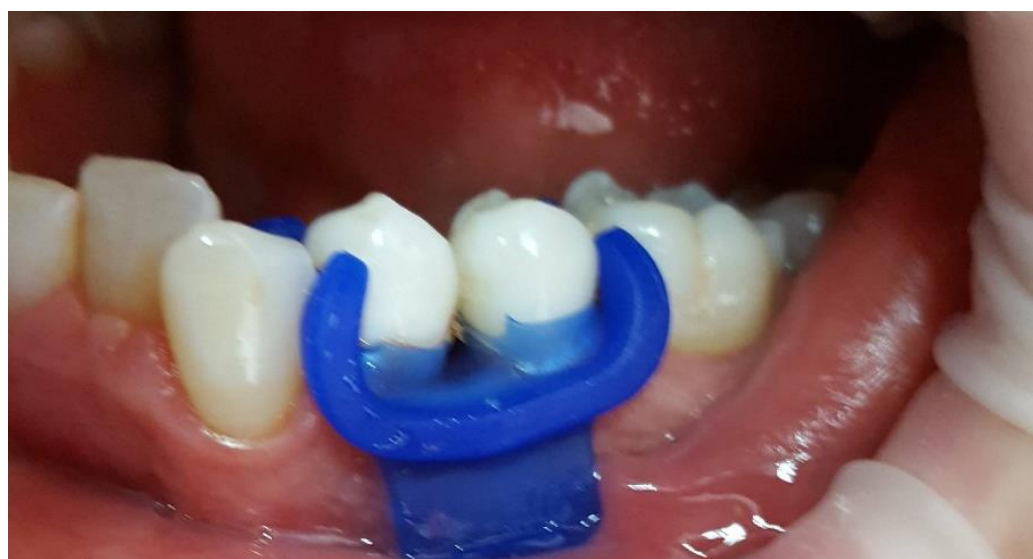

Fig. 18 Final aspect of the infiltrated white spot lesion located on the lower left second premolar 


\section{Conclusion}

An efficient and noninvasive diagnostic and monitoring method of the WSL, associated with infiltration therapy, seems to be the ideal combination which follows the Minimal Intervention Dentistry (MID) principles.

\section{References:}

1. Astvaldsdottir A. et al. (2012). Approximal Caries Detection by DIFOTI: In Vitro Comparison of Diagnostic Accuracy/Efficacy with Film and Digital Radiography. International Journal of Dentistry; (2012), article ID 326401

2. Bilgin G. (2016). Remineralization Potential of Different Agents and Assessment by a New Caries Detection Device. Gavin Journal of Dental Sciences; (2016):39-43

3. Blazejewska A. et al. (2016). Comparison of the Detection of Proximal Caries in Children and Youth Using DIAGNOcam ${ }^{\circledR}$ and Bitewing Radiovisiography. Dental and Medical Problems; 53(4): 468-475

4. Cirligeriu L. et al. (2015). The Importance of Early Diagnosis for Hydroxyapatite Remineralisation in Enamel Caries. Revista de Chimie; (Bucharest); 66(9)

5. Frencken J.E. et al. (2012). Minimal Intervention Dentistry (MID) for managing dental caries - a review. International Dental Journal, 62(5):223-243

6. Gomez J. (2015). Detection and diagnosis of the early caries lesion BMC Oral Health; 15(1):S3

7. Heymann G.C., Grauer D. (2013). A Contemporary Review of White Spot Lesions in Orthodontics. Journal of Esthetic and Restorative Dentistry; 25(2):85-95Tassery H., Manton D.J. (2016). EvidenceBased Caries Prevention, edited by E. Eden; (2):13-20

8. Marinova-Takorovaa M. (2016). Effectiveness of near-infrared transillumination in early caries diagnosis. Biotechnology \& Biotechnological Equipment; 30(6):1207-1211

9. Pagliari Tiano A.V. et al. (2009). Prevalence of enamel white spots and risk factors in children up to 36 months old. Brazilian Oral Research; 23(2):216-22

10. Pitts N.B., Stamm J.W. (2004). International Consensus Workshop on Caries ClinicalTrials (ICW-CCT)-final consensus statements: agreeing where the evidence leads. Journal of Dental Research; 83:C125-128.

11. Russotto F. et al. (2016). Clinical evaluation of near-infrared light transillumination (NIRT) as an interproximal caries detection tool in 
a large sample of patients in a private practice. Journal of Radiology and Imaging; 1(1):1-5

12. Sochtig F. et al (2014). Caries detection and diagnostics with nearinfrared light transillumination: Clinical experiences). Quintessence International; 45(6):531-538

13. Sundararaj D. et al. (2015). Critical evaluation of incidence and prevalence of white spot lesions during fixed orthodontic appliance treatment: A meta-analysis. Journal of International Society of Preventive and Community; 5(6): 433-439.

14. van der Veen M. H. (2015). Detecting Short-Term Changes in the Activity of Caries Lesions with the Aid of New Technologies. Current Oral Health Reports; (2015)2:102-109

15. Walsh L.J., Brostek A.M. (2013) Minimum intervention dentistry principles and objectives. Australian Dental Journal Special Supplement- Special Issue: Minimum Intervention Dentistry.

16. Wojcik-Checinska I. (2015). Specifics of proximal caries and their diagnostics in posterior teeth. Current Issues in Pharmacy and Medical Sciences; 28(2):92-96 